\title{
Aspectos de la perforación de pozos complejos en piedemonte en tiempos de crisis
}

\author{
Abraham Montes $^{1 *}$; Wilson Carreño ${ }^{2}$; Miguel Guío ${ }^{3}$ \\ ${ }^{1}$ MS., Drilling Engineer Drilling \& Completion in Equion-Energía. Bogotá, Colombia. \\ ${ }^{2}$ MS., SWSL Engineer Drilling \& Completion in Equion-Energía. Bogotá, Colombia. \\ ${ }^{3}$ ADT Engineer in Schlumberger. Medellín, Colombia. \\ E-mail: abraham.montes@equion-energia.com
}

\begin{abstract}
Resumen
El agotamiento de los yacimientos convencionales y los retos que implica para la ingeniería son evidentes. En el piedemonte colombiano la tendencia ha sido clara: En los últimos 3 años se han perforado los pozos más profundos con $20808 \mathrm{ft} \mathrm{y} 20300 \mathrm{ft}$. Esta profundidad parece ser demasiado, pero en realidad el número no es suficiente para representar lo grande del reto. Los desarrollos de ingeniería en torno a esta operación y la madurez organizacional alcanzada por la compañía operadora a través de curvas de aprendizaje complejas han reducido significativamente el impacto negativo de estos retos al desempeño de los proyectos. No obstante, a la cantidad de problemas de ingeniería que se requiere manejar, se adicionan las fluctuaciones económicas, políticas y sociales y todas sus causas, tal como la crisis petrolera que inició en 2014. La pregunta que surge es: ¿Cómo perforar pozos profundos en tiempos de crisis?

Las decisiones de mitigación tomadas por gerentes de estrategia implican planeación y ejecución de proyectos optimizando los recursos tanto como sea posible. Esta optimización es un reto más para adicionar a la lista, debido a que su aplicación requiere cuidado especial. Optimización no significa reducir costos a expensas de los beneficios del personal, la calidad o el aseguramiento de los pozos. Significa reducir costos estratégicamente y esto requiere análisis detallados de pozos de correlación y estudio de sensibilidades de las optimizaciones propuestas. Este es la razón por la cual en el piedemonte colombiano los análisis What-if, la capitalización de las lecciones aprendidas y los análisis de riesgos se fundamentan en modelos de planeación robustos. Tal vez perforar a través de capas de carbón ha sido uno de las operaciones más retadoras en el Piedemonte. Sin embargo, la meta ya no es perforar capas de carbón intercaladas o invertidas, es hacerlo en tiempos de crisis.
\end{abstract}

Palabras Clave: Pozos profundos, Tiempos de Crisis, Estrategia, Optimización, Lecciones Aprendidas, Retos.

\section{Aspects of drilling complex wells in foothills in crisis times}

\begin{abstract}
The depletion of conventional reservoirs and the challenges for engineering that this means are not a secret. In Colombian foothills the trend has been clear: In the last 3 years, the deepest wells have been drilled with 20,300 ft and 20,808 ft. This depth could seem to be a lot, but actually the number is not enough to represent how big the challenge is. The engineering development around this operation and the organizational maturity reached by the operator company through complex learning curves, have reduced the negative impacts to the projects' performance. However, to the bunch of engineering issues to handle, it is necessary to add the political, economic and social fluctuations and all its causes, like the oil crisis that started on 2014. Hence, the question is: How to drill deep wells in crisis time?

The mitigation decisions taken by strategy managers imply planning and execution of projects by optimizing the resources as much as possible. Optimization is one more challenge because its application takes special care. Optimization does not mean reducing costs at expense of the personnel's benefits, wells' quality or operations assurance. It means reducing costs strategically and this requires detailed analysis of offset wells and sensibilities of the proposed optimization. This is why in the Colombian foothills, the Whatif analysis, the capitalization of learned lessons and the risks analysis are made on robust planning models. Perhaps drilling through coal beds has been one of the most challenging operations in the foothills. Anyway, now the goal is not to drill through interbedded or inverted coal layers anymore. It is doing it in crisis time.
\end{abstract}

Keywords: Deep wells, Crisis time, Strategy, Optimization, Learned Lessons, Challenges.

Cita: Montes, A., Carreño, W. y Guío, M. (2018). Aspectos de perforación de pozos complejos en piedemonte en tiempos de crisis. Revista Fuentes: El reventón energético, 16(1), 87-97. 


\section{Introducción}

La reducción del precio del crudo tiene grandes consecuencias para los países cuya economía se ha desarrollado con bases petróleo-dependientes. Los grados de afectación y de petróleo-dependencia guardan relación directa. El precio del crudo tiene tantas variables en función que la incertidumbre de las proyecciones puede ser abismal. Es por esto que una consecuencia inmediata, como en cualquier industria, es que las compañías operadoras pierdan el interés por la ejecución de proyectos cuya viabilidad aparece en precios atractivos del crudo. En la gráfica a continuación, se muestra el número de taladros de perforación en operación en Colombia para los años 2015 y 2016 (CAMPETROL, 2016).

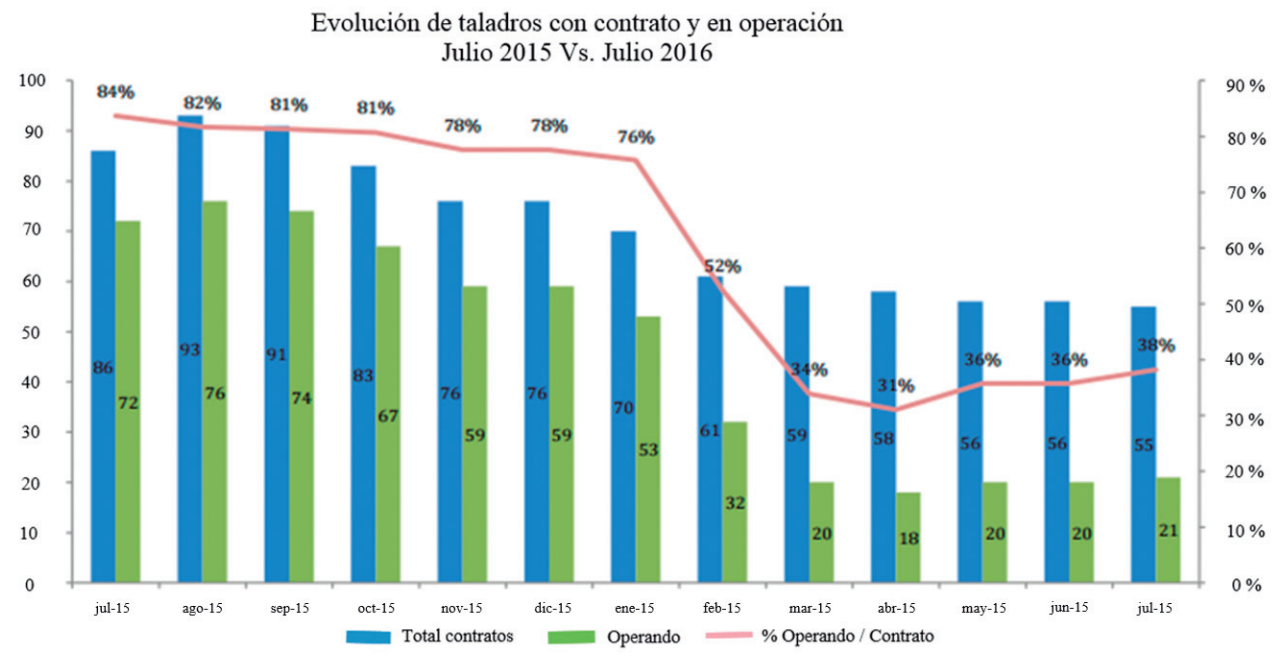

Figura 1. Evolución de taladros con contrato y en operación Julio 2015 Vs. Julio 2016

De lo anterior se derivan una cantidad de problemas económico-sociales tales como la reducción crítica del aporte al PIB (Producto Interno Bruto) y el aumento del desempleo (Barriga, 2009) (Banco de la República de Colombia, 2016).

En los pozos perforados en el Piedemonte, los costos amenazan con márgenes muy cortos para comprometer la viabilidad de los proyectos. Es por esto que en la planeación y ejecución se han desarrollado estrategias para apuntar a soluciones de ingeniería que permitan reducir los costos asociados a factores clave (Ayala, Torres, Valencia \& Loaiza, 2016). Algunos de estos factores clave son: formaciones muy duras y abrasivas (Ayala, Gómez \& León, 2011), incertidumbre geológica (Portilla, Suárez \& Corzo, 2012), estabilidad de pozos (Ayala, Benítez \& Valencia, 2017), calidad de hueco (Campana \& Tapia, 2017) y viajes de tubería, manejo social, entre otros.

\section{Soluciones detrás de las complejidades de pozos en el piedemonte}

A continuación, algunas de las mayores complejidades del Piedemonte, los incidentes operacionales que se han generado y las estrategias de mitigación.

\section{Formaciones duras y abrasivas}

Las formaciones duras y abrasivas que se atraviesan en la perforación de los pozos, algunas de las cuales no son de interés, pueden llegar hasta los $800 \mathrm{ft}$ de espesor por las trayectorias de los pozos y la incertidumbre en cuanto a los buzamientos. El reto se convierte en lograr corridas largas y a buena ROP (Rate of penetration), lo cual reduce la cantidad de viajes de tubería. Algunas de las pocas corridas que ejemplifican esta complejidad en los últimos tres años.

Tabla 1. Brocas corridas hechas en formaciones duras y abrasivas

\begin{tabular}{llccc}
\multicolumn{1}{c}{ Broca } & \multicolumn{1}{c}{ Tipo de Motor } & Corrida & ROP Efectiva & \multicolumn{1}{c}{ Desgaste } \\
\hline Impregnada & Motor de Altas revoluciones & $55 \mathrm{ft}$ & $4.2 \mathrm{ft} / \mathrm{h}$ & $8-8-W T-A-X-1-N O-P R$ \\
Tricónica de insertos & Sin motor de fondo & $107 \mathrm{ft}$ & $2.7 \mathrm{ft} / \mathrm{h}$ & 2-6-BT-G-E-I-WT-HR \\
PDC 8 blades / $16 \mathrm{~mm}$ & Rotary Steerable System & $57 \mathrm{ft}$ & $2.2 \mathrm{ft} / \mathrm{h}$ & $6-4-$ O-N-X-I-BT-PR \\
PDC 8 blades / $13 \mathrm{~mm}$ & Sin motor de fondo & $85 \mathrm{ft}$ & $2.2 \mathrm{ft} / \mathrm{h}$ & $6-8-$ RO-G-X-14-BT-PP \\
PDC 8 blades / 13 mm & Sin motor de fondo & $127 \mathrm{ft}$ & $2.4 \mathrm{ft} / \mathrm{h}$ & $5-8-L T-A-X-1-L M-P P$ \\
\hline
\end{tabular}


La estrategia para este factor se ha centrado en impulsar el $\mathrm{I}+\mathrm{D}+\mathrm{i}$ (Investigación, desarrollo e innovación) en conjunto con las compañías de servicios a través de la apertura a nuevas tecnologías. Entre estas tecnologías, podrían mencionarse: corridas con turbinas y brocas impregnadas de buen volumen matricial, corrida de brocas PDC con cortadores rotarios y corridas con MPD (Brunton, et al., 2014) (Besson, et al., 2000) (Bone, et al., 2013).

A manera informativa, la siguiente tabla ilustra los beneficios económicos logrados en promedio en la perforación de formaciones abrasivas. En este caso, se toma en consideración un espesor aparente de 600 $\mathrm{ft}$ de la formación Mirador, perforada con brocas de $81 / 2$, compuesta en general por arenas muy finas y limpias.

Tabla 2. Comparación de corridas realizadas (Promedio) para $600 \mathrm{ft}$ de espesor aparente de la formación Mirador en un tamaño de 8 1/2".

\begin{tabular}{lcc} 
Medida & $\begin{array}{c}\text { Pozos antes } \\
\text { de 2013 }\end{array}$ & $\begin{array}{c}\text { Corridas Brocas } \\
\text { ONYX 360 }\end{array}$ \\
\hline Número de brocas corridas & 6 & 3 \\
Corridas promedio & $109 \mathrm{ft}$ & $225 \mathrm{ft}$ \\
Horas de Circulación & $430 \mathrm{~h}$ & $140 \mathrm{~h}$ \\
ROP Promedio & $3.1 \mathrm{ft} / \mathrm{h}$ & $3.5 \mathrm{ft} / \mathrm{h}$ \\
ILT por Vibraciones & $21.8 \mathrm{~h}$ & $2.9 \mathrm{~h}$ \\
Costo Promedio & $\$ 4,680,000$ & $\$ 2,610,000$ \\
\hline
\end{tabular}

En términos generales, se estima que, en promedio, la estrategia agresiva de uso de nuevas tecnologías para la perforación de formaciones abrasivas ha capitalizado un $30-40 \%$ de mejoramiento continuo para la compañía, traducido en ahorros y mejora de la eficiencia.

\section{Incertidumbre geológica}

Es difícil cuantificar esta complejidad debido a la gran cantidad de tiempos no planeados que pueden generarse a partir de la incertidumbre geológica. No obstante, para introducir este factor, se muestra una sección transversal característica del piedemonte en la figura 2. Es evidente la complejidad tectónica del sector, donde las capas se han plegado, fracturado y fallado; haciendo que perforarlas tenga una gran incertidumbre. Dentro de las consecuencias más relevantes a partir de la incertidumbre geológica, las que más han causado impactos son las siguientes:

Desfase de los buzamientos planeados. Aunque se han tomado registros de rayos gamma azimutal, se encuentra aún algunos casos de alto desfase entre el buzamiento planeado y el buzamiento aparente encontrado. La consecuencia inmediata de esto es que una formación que se planee perforar con un espesor, puede resultar siendo el doble, puede desaparecer o ser reemplazada por otra. En cualquiera de los casos, si el resultado es la perforación de una capa dura y abrasiva no planeada de por ejemplo $300 \mathrm{ft}$, se estarían generando en promedio 6 días de tiempo no planeado, con un impacto en costo de USD \$ 790K USD.

Yacimientos perdidos. Yacimientos que originalmente se plantearon en la prognosis geológica y que fueron tomados dentro de la producción planeada del pozo, pueden no ser encontrados. En estos casos, podría llegarse a perder una cantidad considerable de producción. No obstante, cabe aclarar que también se ha dado el caso contrario: Formaciones de interés que no estaban en el plan, han sido encontradas, incrementando la producción planeada del pozo.

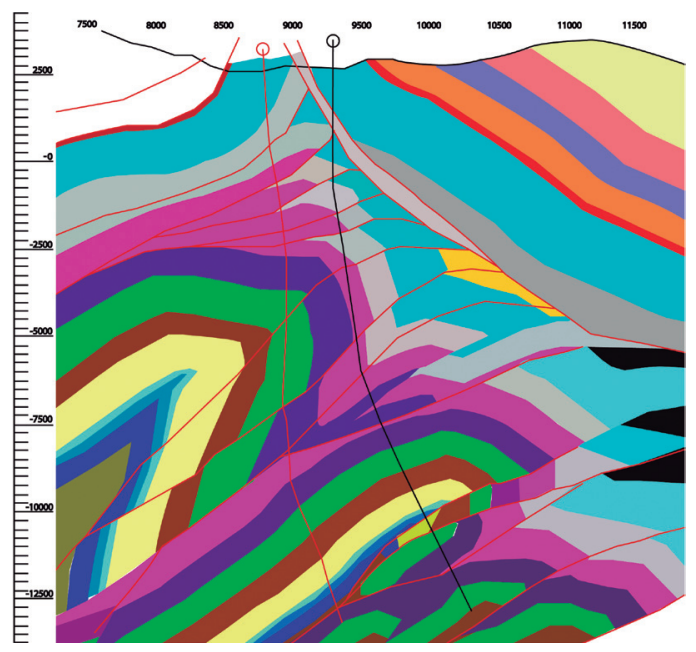

Figura 2. Sección transversal típica del Piedemonte

Revestimientos mal declarados. Debido a la interpretación errónea de un tope de formación, puede declararse el punto de revestimiento en una profundidad inadecuada.

Caso 1: Mientras se perfora la sección de 14 3/4" con un fluido de $11.7 \mathrm{ppg}$, cuyo punto de revestimiento está planeado en el tope de una formación depletada con presión de poro de $8.5 \mathrm{ppg}$; debido a la incertidumbre geológica, se encuentra el tope de la formación 100 $\mathrm{ft}$ antes de lo esperado. Al entrar en dicha formación con un MW (Mud Weight) tan alto, se inducen pérdidas masivas. Automáticamente la presión de circulación cae 400 psi, el peso de la sarta disminuye y se pierde el retorno. Al perderse el soporte para los cortes en el 
anular, estos caen sobre las herramientas del BHA, creando un empaquetamiento. La reacción inmediata del perforador es intentar levantar la sarta, ante lo cual se encuentra con un evento de stall out y sobretensión. Este caso, en el cual se presentó pega de tubería y pérdidas masivas de circulación, resulta en sidetrack del pozo.

Caso 2: Mientras se perfora una formación inestable con un fluido cargado en asfaltos, se espera encontrar el tope de una formación de interés. En este tope se bajará el revestimiento con el fin de perforarla luego con un fluido libre de asfaltos para reducir el daño de formación. Se encuentra un intervalo arenoso $650 \mathrm{ft}$ antes de lo planeado, donde la ROP cae drásticamente. En este punto no se tiene pérdidas de fluido a pesar de perforar con un sobre-balance significativo. En este escenario, ante la ambigüedad de los parámetros, se decide llamar punto de revestimiento. Luego, en efecto se perforan $100 \mathrm{ft}$ (Se esperaban $500 \mathrm{ft}$ ) de la formación yacimiento y se encuentra nuevamente la formación inestable anterior, la cual fue perforada con un lodo de muy bajo peso y sin concentración de asfaltos. Como resultado, la inestabilidad de esta formación causó 4 eventos de empaquetamiento y tiempos de viaje exagerados, con un impacto en costo de aproximadamente 1.5 MM USD.

Litologías intercaladas no previstas. La variación constante de las litologías ha sido causante de muchos problemas tales como vibraciones de la sarta en altos niveles, desgastes prematuros de las brocas, bajas ROP, entre otros. Las vibraciones pueden producir una caída sustancial de la ROP promedio, debido a la cantidad de veces que se hace necesario levantar la sarta para controlarlas. La operación de una sarta con vibraciones incontroladas puede sufrir daños severos de integridad. Una broca prematuramente dañada no solamente genera viajes adicionales; también pone en riesgo la integridad de la siguiente corrida en caso de que la broca haya quedado fuera de calibre o haya perdido cortadores que hayan quedado en fondo.

Inestabilidad del pozo. La incertidumbre geológica lleva también a que los modelos de estabilidad de pozos sean reevaluados constantemente durante la perforación, ya que se encuentra que, en muchas ocasiones, el peso del lodo es inadecuado para prevenir los derrumbes excesivos de las paredes de los pozos. El MW se convierte en una herramienta de precisión en términos de estabilidad. Estar por debajo o por encima del MW requerido, aumenta la tasa de derrumbes considerablemente bien sea por morfologías tipo astillas o tipo tablas y bloques. Como consecuencia de perforar con un MW que podría no ser el adecuado, ha generado múltiples eventos de empaquetamiento, muchos de los cuales han terminado en sidetracks. Dependiendo de la sección, un evento de este tipo puede costar alrededor de 5 MM USD.

Estructuras imprevistas. Es muy frecuente encontrar zonas extremadamente falladas o fracturadas, las cuales no son contempladas en la planeación. Estas zonas se caracterizan por la generación de grandes cantidades de derrumbes y pérdidas de circulación considerables. A manera de ejemplo, se han tenido casos de $15 \mathrm{Bls} / \mathrm{h}$ de derrumbes en un hueco de $81 / 2$ " que resultan en empaquetamientos. Por otro lado, también hay casos de pérdidas masivas que han resultado en pegas diferenciales. Para ambos ejemplos, huecos perdidos que requieren sidetrack del pozo.

Los costos asociados a problemas generados por incertidumbre geológica pueden ser un porcentaje considerable del presupuesto aprobado del proyecto. No obstante, se han tomado muchas medidas para atacar este problema. Algunas de ellas, han logrado disminuir dicho impacto económico en cierta medida. A continuación, se muestra a manera de ilustración, la reducción en los costos asociados a incertidumbre geológica, logrado con el uso de nuevas tecnologías en el piedemonte.

Corridas de GR Azimutal o UBI: Logran dar un mayor entendimiento del estado geomecánico del pozo, es decir la dirección de las fracturas, la presencia de breakouts, el buzamiento de las capas, la reevaluación de los modelos de estabilidad y las prognosis geológicas, entre otros. Es importante tener en cuenta que estas corridas no siempre son posibles aun usando herramientas LWD, dada la condición del hueco. Además, tienen un costo elevado (Madjidi, 2001) (Schlumberger, 2002).

Brocas Híbridas: Las brocas que combinan los mecanismos de corte de los distintos tipos de brocas pueden resultar muy versátiles y lograr muy buenas corridas en formaciones intercaladas. Considere una formación muy heterogénea y con intercalaciones frecuentes como Los Cuervos en el Piedemonte. Correr una broca de conos cuyo mecanismo de corte es el grinding, haría la perforación muy lenta o imposible para intervalos plásticos arcillosos. Por otro lado, correr una broca PDC con cortadores de $13 \mathrm{~mm}$ lograría muy buenas ROP en intervalos arenosos, pero ROP pobres en intervalos plásticos. El uso de una broca 
híbrida como la Kymera (Baker Hughes), permite combinar ambos mecanismos de corte (Triturado y cizalla), logrando buenas ROP en ambas litologías. Particularmente en la formación Los Cuervos, se han logrado corridas de hasta $800 \mathrm{ft}$ con ROP (Con conexiones) de hasta $15 \mathrm{ft} / \mathrm{h}$ en promedio para huecos de 10 5/8” (Rickard, Bailey, Pahler y Cory, 2014).

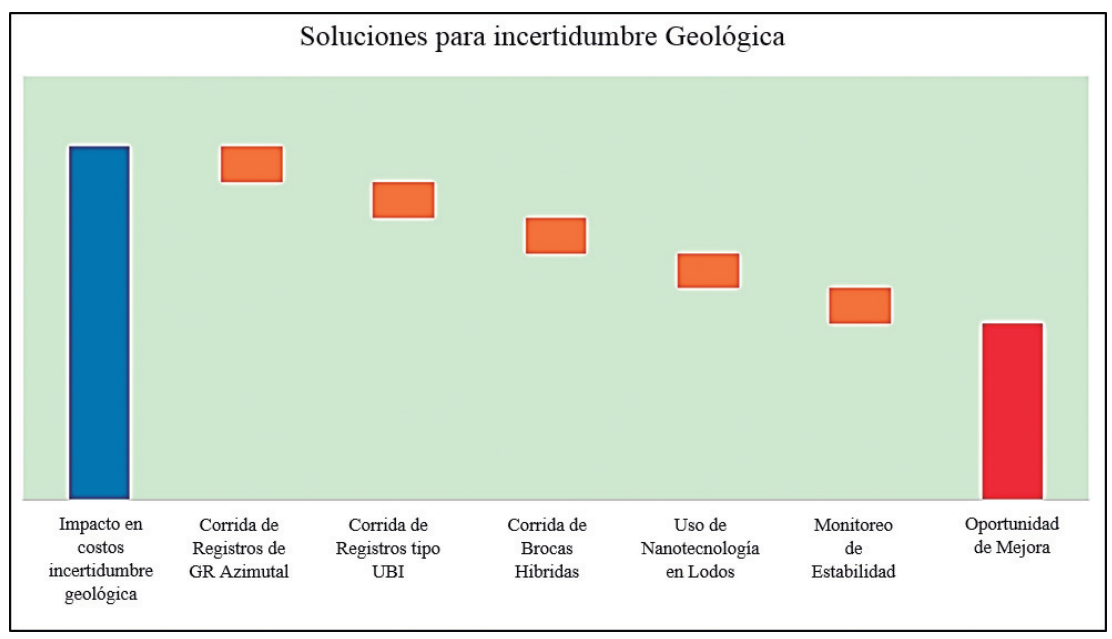

Figura 3. Tecnologías usadas en la disminución de la incertidumbre geológica.

Estrategias del fluido de perforación: Las claves en cuanto a desarrollo del fluido de perforación han sido los asfaltos, la nanotecnología y la distribución adecuada de tamaños de partícula. Se ha encontrado que hay una disminución en al menos el 50\% de los costos asociados a pérdidas de fluido gracias al uso de estos elementos para puentear las fracturas naturales y las gargantas porales de las formaciones permeables.

Monitoreo de Estabilidad: El análisis de los derrumbes en cuanto al volumen, morfología y tamaños, en conjunto con el monitoreo del ECD con sensores de presión anular, han logrado tomar decisiones oportunas de variar el peso del lodo, incrementar el material de puenteo o incluso circular el pozo y mitigar la posibilidad de empaquetamientos de la sarta (Walt, et al., 1998).

\section{Formaciones frágiles}

En el piedemonte, las estructuras están compuestas en su parte superior por formaciones intercaladas arenosas y arcillosas con abundantes mantos de carbón. La complejidad de perforar carbones consiste en: Fragilidad, tendencia a cerrar, susceptibilidad a invasión del lodo, altos contenidos de gas, entre otros. Escoger un peso de lodo inadecuado o seguir procedimientos incorrectos, puede causar una gran cantidad de problemas relacionados con los carbones y generar desviaciones considerables del plan. Recientemente en el piedemonte, se perforó un pozo cuya sección de 14 3/4" atravesó numerosos mantos de carbón. Mientras se viajaba el hueco abierto, se obtenían ratas de derrumbes exageradas de hasta $15 \mathrm{Bbl} / \mathrm{h}$. Se cree que el sobre balance excesivo, sugerido para estabilizar las arcillas, causó la invasión y posterior inestabilidad de los mantos de carbón, estos cayeron en numerosas ocasiones al BHA, generando su atrapamiento. Además, los fragmentos de carbón que el fluido era capaz de transportar a superficie eran de hasta $18 \mathrm{~cm}$, por lo cual se cree que la sarta fue atrapada en numerosas ocasiones por carbones de mayor tamaño y agregados de arcillas inestables. Como resultado de esto, se tomó la decisión de realizar sidetrack del hueco en 3 ocasiones.

Perdiendo 2 sartas y logrando recuperar 1 mediante el uso del martillo. Adicional a esto, fue necesario un plan completo para reanudar el pozo de manera segura. En total, el sobrecosto está alrededor de $20 \mathrm{MM}$ Usd. A manera de ejemplo, considere el FEL del hueco abierto de uno de los sidetracks del pozo mencionado (Figura 4).

Algunas estrategias utilizadas en términos de ingeniería y operaciones para perforar estos intervalos recomendados a partir de todas las lecciones aprendidas podrían resumirse de la siguiente manera:

Reevaluación constante del modelo de estabilidad: Los volúmenes de derrumbes que se obtienen deben ser tomados en consideración para reevaluar de forma constante el WSM (Wellbore Stability Model) 
y de esta forma reaccionar oportunamente para los cambios de MW que requiera el pozo. Reaccionar tardíamente podría aumentar el tiempo de exposición de las formaciones al lodo y por tanto aumentar la inestabilidad y envejecimiento del hueco.

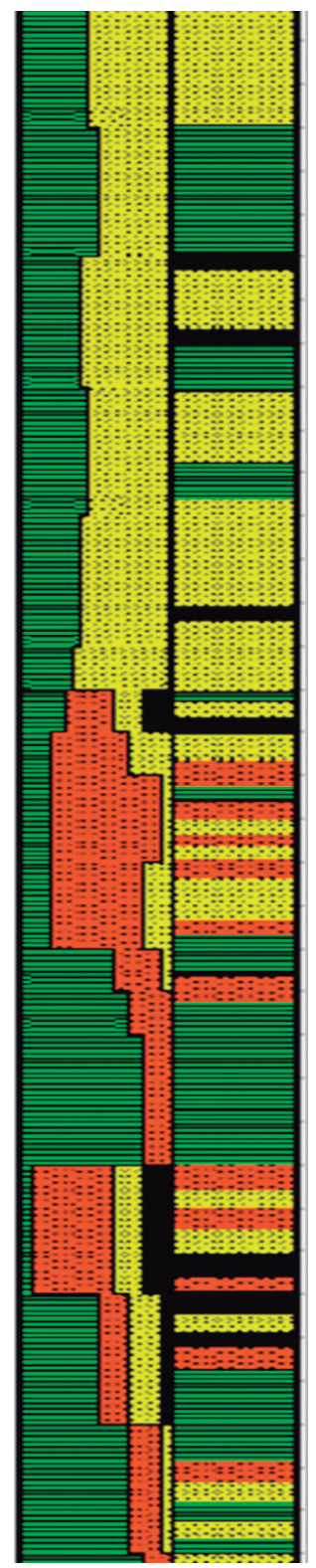

Figura 4. FEL Con abundantes Mantos de carbón

Pulsos de Presión: Se encontró que los pulsos de presión generados por el envío de comandos a la herramienta direccional o por la puesta y la quitada de las bombas de forma brusca, aumentan el riesgo de inestabilidad de los carbones. Como resultado de este análisis, entre otras cosas se tomó la decisión de obtener los surveys mediante la variación de la rotación, sin variar el caudal de bombeo.
Repaso cauteloso de las paradas: Cada media parada perforada, debe ser 2 veces repasada usando altas revoluciones para evitar eventos de stall out y aumentar la posibilidad de triturar los fragmentos grandes de carbones que puedan caer. Una vez repasada, se acondiciona la zona de carbones y se disminuye la posibilidad de que cierren sobre la sarta y sus fragmentos caigan al BHA.

\section{Orden Social}

Cada vez es más frecuente que las comunidades vean la presencia de las compañías operadoras como sustitutos del deber constitucional del estado, exigiendo que estas se hagan cargo de las necesidades no satisfechas o se enfrenten a la permanente amenaza de paros y bloqueos en las zonas de influencia que terminan encareciendo algunos proyectos y haciendo inviables otros (Gutiérrez, 2016). El costo de este factor puede llegar a alcanzar hasta el $20 \%$ de los presupuestos. Para un proyecto cuya operación es detenida por protesta durante 15 días, tiene una desviación promedio de 2.0 MM USD. No obstante, en el piedemonte se ha mantenido la política de trabajar en beneficio de la comunidad: Las obras sociales y el fomento del buen trato.

\section{Dirección estratégica de proyectos de perforación}

A diferencia de la mayoría de las industrias, la industria del petróleo es mucho más sensible a parámetros más susceptibles de la comunidad mundial. Como resultado, las compañías petroleras deben realizar planes muy enfocados desde la dirección estratégica que permitan conferirle a sus operaciones la flexibilidad necesaria para sobrevivir en este mercado.

Para las operaciones de perforación en particular, hay algunas estrategias que marcan la diferencia. Algunas de ellas son:

Gestión eficaz del recurso humano: Existe un camino errado tomado con frecuencia desde la gerencia estratégica en cuanto al ahorro a manos de beneficios para el recurso humano. La clave está en entender que dichos beneficios son en realidad una inversión. En épocas de crisis, la incertidumbre se convierte en un factor de riesgo ya que no solamente las situaciones personales y económicas del equipo de trabajo se ven afectadas, también el futuro de la industria misma. Es por esto que existe una pérdida de concentración y foco natural. Contra esta situación, existen dos armas fundamentales que desde luego tienen ganancias que son incluso tangibles al corto plazo (Maella, 2010) (Lopez y Hernández, 2013): 
- Motivación: Es una forma de contrarrestar la pérdida de foco natural que se da en momentos de crisis económica o fluctuación desfavorable de la industria. Un equipo de trabajo motivado, tiene mayor concentración, por ende, es un equipo menos susceptible a sufrir incidentes, tiene mayor rendimiento operacional, opera con mayor eficacia y eficiencia, aumenta su sentido de pertenencia por el proyecto e incrementa su participación en los procesos de mejora continua.

- Reconocimiento: Un complemento fundamental del punto anterior. El reconocimiento incluso puede ser una herramienta con mayor potencial. Un equipo de trabajo al cual se le ha reconocido públicamente y se le ha premiado por una excelente labor, dejando claro los beneficios que han logrado para la compañía por su trabajo eficiente, generan un ambiente colaborativo y participativo invaluable para la compañía. Además, promueve el mejoramiento continuo y establece metas cada vez más ambiciosas para los equipos.

Como ilustración del caso anterior, considere una de las operaciones dentro de la perforación que consumen mucho tiempo no efectivo: Los viajes de tubería. Dentro del hueco revestido, la optimización de los viajes es fundamental para reducir al mínimo los tiempos no efectivos de perforación. A continuación, se muestra las velocidades de viaje para la sección de 10 5/8" para cierto pozo, en el cual se ha implementado el reconocimiento público para los equipos de trabajo conforme ha progresado la operación:

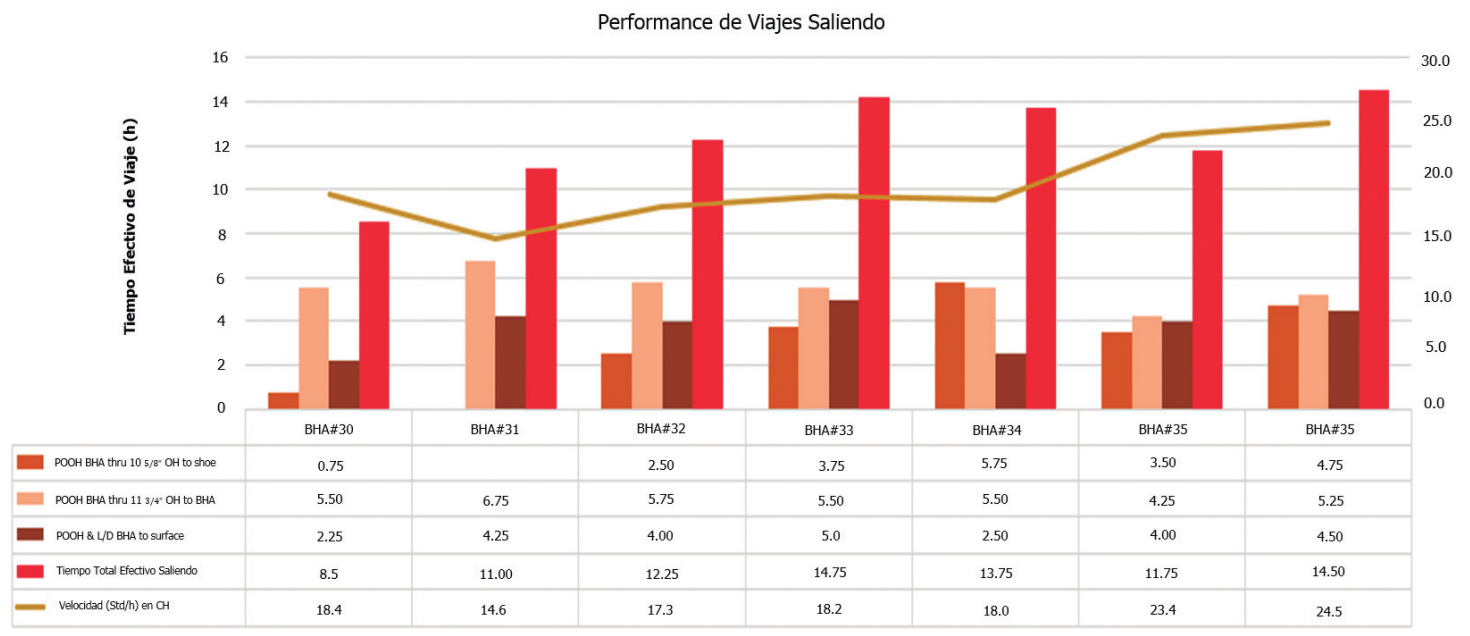

Figura 5. Rendimiento de viajes en sección de 10 5/8”

Como puede observarse, las cuadrillas se han trazado metas cada vez más ambiciosas y han logrado llegar cerca a las 25 paradas / hora para el hueco revestido de $113 / 4$ ". Para este revestimiento de aproximadamente $10.000 \mathrm{ft}$, un viaje hecho a 25 paradas por hora frente a uno hecho a 15 paradas por hora, como inició el proceso significa un ahorro de aproximadamente $3 \mathrm{hrs,}$ o lo que es equivalente $25 \mathrm{~K}$ Usd.

Intensificación del Mejoramiento Continuo: El mejoramiento continuo no solamente debe tomarse como una herramienta para alcanzar mayores niveles de madurez organizacional, mediante la capitalización de las lecciones aprendidas y el ambiente totalmente propositivo y de trabajo colaborativo. También es una herramienta para afrontar tiempos de crisis, en la medida en que las ideas de mejora continua son finalmente traducidas en su mayoría a mejoramiento de la eficiencia, mejoramiento de las condiciones de trabajo o incluso operaciones sustitutivas que significan grandes ahorros para la compañía (García, Quispe y Ráez, 2003) (Project Management Institute, 2013) (Grant, 2006). No obstante, el mejoramiento continuo necesita de un direccionamiento estratégico en épocas de crisis, con el fin de no caer en la estabilidad indeseable de un programa o una campaña empresarial, sino por el contrario mantenerse en la dinámica creciente que se busca. Para lograr estos objetivos, es necesario:

- Usar la motivación y el reconocimiento como pilares fundamentales del mejoramiento continuo. El reconocimiento es incluso mandatorio, ya que eliminarlo, llevaría a la congelación inmediata del crecimiento organizacional. 
- Mantener un ambiente propositivo y participativo, incrementando el sentido de pertenencia de la compañía y de los proyectos.

- Enfocar el programa hacia ideas que se traduzcan en ahorros tangibles o intangibles. No necesariamente una mejora se traduce en un ahorro económico e inmediato. Es posible que la cultura empresarial juegue un papel fundamental en la focalización del mejoramiento continuo. Un ejemplo claro es el mejoramiento de las condiciones de seguridad o ambientales, lo cual puede ser prioritario para una compañía cuyo valor fundamental sea el valor por la vida y la seguridad.

- Promover horizontalidad dentro de los equipos de trabajo. Los equipos de trabajo cuya estructura es demasiado piramidal o vertical, tienden a enfriar sus plataformas de madurez, dejando de un lado el mejoramiento continuo o haciéndolo muy lento.

A manera de ilustración, en los últimos 3 años, el enfoque que se ha logrado dar al mejoramiento continuo, ha logrado los mayores ahorros operacionales, tales como:

- Armes y desarmes de ensamblajes de fondo en tiempos muertos: Usando el hueco del ratón, los winches hidráulicos de la mesa del taladro y la llave hidráulica, es posible realizar los armes y desarmes de herramientas como HWDP y DC mientras se perfora o mientras se circula. Con esto, en un viaje redondo puede llegar a ahorrarse hasta 4 horas de operación o lo que es equivalente: $30 \mathrm{~K}$ Usd. Para una sección en la cual sea necesario realizar 5 viajes redondos con cambio de ensamblaje, el monto ahorrado sería de $150 \mathrm{~K}$ Usd.

- Perforación de formaciones abrasivas con brocas Onyx 360 (Smith): Se ha logrado tener corridas muy largas $(250 \mathrm{ft})$, con buenos rendimientos $(3-5$ $\mathrm{ft} / \mathrm{h}$ ) y con buena condición del hueco (En calibre). Para una formación abrasiva que se espera de $500 \mathrm{ft}$ de espesor aparente, planeada posiblemente con 4 corridas, puede lograrse reducir hasta el $40 \%$ de los costos.

- Uso de herramientas direccionales tipo RSS sin empaquetaduras en pads: Es muy común que las brocas deban ser sacadas de fondo por falla de las herramientas direccionales. En el caso de los RSS como el PowerDriveX6 (Schlumberger), se tiene una limitación en cuanto a las horas de circulación debido a la posible pérdida de integridad de los sellos que permiten los kicks de los pads que realizan el trabajo direccional. Para un tamaño de herramienta de 8", se han logrado corridas de hasta $150 \mathrm{hrs}$ sin problemas en los sellos. No obstante, la limitación en horas de rotación para el BHA de este tamaño, es cerca de 250 hrs. Por lo tanto, es posible que en promedio se pierdan $100 \mathrm{hrs}$ de rotación por falla de la herramienta direccional. El uso de pads impulsados por esferas como el Orbit (Schlumberger), elimina esta limitación y aumenta considerablemente la posibilidad de usar todas las horas disponibles para el BHA y de esta manera reducir los viajes necesarios hasta en 50\%.

Eliminación de la sobre-ingeniería: La sobreingeniería es un factor considerable en el aumento de costos de un proyecto. No obstante, la optimización de estos diseños que puedan contener sobre-ingeniería, requieren estudios detallados y debidamente soportados con la experiencia, con el fin de no caer en la disminución del aseguramiento. Trabajar en eliminar la sobre-ingeniería no equivale a disminuir la calidad, escatimar la seguridad o ablandar las políticas dadas a partir de la cultura empresarial y establecer ese límite requiere de asignación de recursos exclusivos para estudios detallados o consultorías. Algunos de los detalles de sobre- ingeniería que se han eliminado en Piedemonte por ejemplo son:

- Uso de desviador en locaciones con pozos previos: Los desviadores usados en la primera sección, como se conoce tienen el fin de permitir la quema de gas superficial lejos del equipo de perforación. No obstante, en locaciones donde se han perforado otros pozos, la probabilidad de encontrar gas superficial es muy baja. Considerando esto, se determina como una medida innecesaria el uso de un desviador, cuyo montaje requiere un tiempo operacional considerable y además un costo de renta elevado.

- Uso de tubería grado 110TT para revestimiento: La tubería TT y la tubería $\mathrm{T}$ difieren en el grado de aseguramiento que se da al espesor de pared y por tanto a los rangos permitidos de trabajo en cuanto a colapso y estallido. Es posible trabajar con tubería $\mathrm{T}$ en intervalos donde no se requiera tanta exigencia, realizando nivelación de los materiales a utilizar para las corridas de revestimiento.

- Sobre-Centralización de Revestimientos: Mediante simulaciones detalladas de la posición de la sarta de revestimiento una vez alcanza el fondo 
y los intervalos de interés, es posible re-definir la cantidad de centralizadores a correr, priorizando así los intervalos que puedan ser posibles yacimientos y eliminando o reduciendo sustancialmente los intervalos que no son de interés. La ganancia principal, no está en los protectores mismos, sino en la facilidad para llegar al fondo, ya que los protectores confieren rigidez adicional a la sarta y aumentan la probabilidad de encontrar restricciones durante la corrida.

Mejoramiento de la Eficiencia: La pérdida de la eficiencia puede darse en todos los eslabones de la cadena de valor en un proyecto de perforación. La manifestación más frecuente de este fenómeno es la duplicidad de trabajo. A continuación, se mencionan algunos ejemplos de falta de eficiencia en proyectos de perforación en Piedemonte:

- Investigaciones ineficaces: Los incidentes de cualquier índole requieren ser investigados con el fin de determinar su causalidad y generar un plan de acción para mitigar dichas causas. Es posible que cuando no se realiza una investigación eficaz, las causas raíces encontradas de cierto evento no sean las correctas. En este escenario, se tomarán las medidas de mitigación erróneas y no se atacará la causa raíz del evento principal. Ante esta situación, el riesgo continuará inminente hasta generar un nuevo incidente cuya fuente yace en el mismo riesgo no detectado. Además de las consecuencias inmediatas del incidente, también se generará nuevamente un trabajo investigativo para determinar la causalidad, con lo cual se habrá perdido mucho trabajo y se habrán tenido 2 incidentes. En piedemonte, se ha intensificado la capacitación en investigación de incidentes y se ha hecho hincapié en la necesidad de realizar investigaciones eficaces con el fin de capitalizar las lecciones aprendidas y promover el crecimiento organizacional.

- Pérdida de sinergias internas: Las sinergias pueden representar ahorros importantes si se identifican e implementan oportunamente. Un ejemplo de esto es el manejo de pérdidas de circulación o el acondicionamiento del fluido de perforación con material de pérdida. Es común que esto se haga usando carbonato de calcio de distinta granulometría de acuerdo a un PSD establecido. No obstante, la pérdida de la sinergia entre la ingeniería de fluidos y el control de sólidos, puede generar que el material agregado para artillar el fluido, luego se descarte en el sistema de zarandas o en los hidrociclones, generando la necesidad de agregar nuevamente el material para ajustar las concentraciones requeridas y por ende generando un sobrecosto para el proyecto. En el piedemonte se han tomado varias estrategias para promover la sinergia continua en los equipos de trabajo. Una de ellas es el uso de servicios integrados, con el cual las líneas de operación son de una misma compañía, eliminando así tensiones generadas por el mercado y garantizando una misma plataforma de estándares y cultura empresarial. Por otro lado, se incentiva el trabajo en equipo constantemente y se generan las condiciones suficientes de comunicación y concertación en el equipo de trabajo.

- Pobre Protección en Gauge de las Brocas: Al perforar una formación abrasiva, es común que las brocas con pobre protección en el área del gauge, pierdan su calibre y perforen un hueco de menor tamaño al esperado por un intervalo. Como consecuencia, la siguiente broca debe acondicionar dicho intervalo que ha quedado fuera de calibre hasta el tamaño correcto, operación que tardará un tiempo importante y causará un desgaste a la broca antes de llegar a fondo, es decir se iniciará la broca con un desgaste previo. Como medida de mitigación de este problema, se ha diseñado por parte de las compañías de servicio una protección al gauge muy superior, que asegura que los huecos quedarán en calibre.

- Gestión Documental Ineficiente: Ocurre cuando se duplica la información, haciéndola ambigua o errónea y gastando recursos innecesarios. Un ejemplo sucede cuando se solicita desde la gerencia análisis de información que ya han sido realizados anticipadamente, pero que se han perdido por una gestión documental ineficiente. La consecuencia de este problema se manifiesta en la lentitud de evolución empresarial, debido a que la mayoría de los recursos se utilizan ineficientemente para realizar el mismo trabajo, en vez de actuar prospectivamente. En tiempos de crisis, se requiere que las compañías sean muy dinámicas y este problema confiere rigidez y estabilidad. Para eliminar este problema, una de las estrategias usadas consiste en la unificación de toda la información a través de un software de manejo de información sofisticado que permita obtener información a todos los niveles (Estratégico, táctico u operativo) al instante de requerirla, desde una misma fuente. 


\section{CONCLUSIONES}

Ante proyectos con riesgos tan complejos como los pozos perforados en el Piedemonte Colombiano, es necesario establecer estrategias que permitan a la compañía adquirir la flexibilidad necesaria para resistir fluctuaciones imprevisibles de la industria y aprovechar dichas situaciones para adquirir madurez y continuar creciendo dentro de la dinámica del mejoramiento continuo, sin caer en el congelamiento de ideas, la jerarquización de la estructura organizacional o la decadencia de la política empresarial. Dentro de las estrategias fundamentales se destacan:

1. Análisis de causa raíz eficaz para problemas operacionales o de cualquier índole para tomar medidas de mitigación acertadas.

2. Aprovechamiento del tiempo de crisis para enfocarse hacia el planteamiento de proyectos que tomarán forma en el futuro de acuerdo a la fluctuación del mercado y la industria.

3. Enfoque hacia el mejoramiento continuo como fuente principal de ahorros, generando un ambiente participativo y promoviendo la investigación y el desarrollo en beneficio de la operación.

4. Gestión estratégica del recurso humano para operar de forma segura y eficiente en tiempos de crisis.

5. Eliminación de la ineficiencia en todas las áreas de la compañía.

La clave está en entender la crisis como una oportunidad y generar el ambiente propicio para que se aproveche dicha oportunidad en beneficio de la compañía.

\section{REFERENCIAS}

1. Ayala, D., Benítez, A., \& Valencia, R. (2017). Optimización de la Tasa de Penetración mediante el análisis de las vibraciones al perforar, caso de estudio Ecuador. Revista Fuentes, 15(1), 27-40.

2. Ayala, D., Torres, H., Valencia, R., \& Loaiza, M. (2016). Impacto del Tiempo no Productivo en operaciones de perforación y análisis de los datos mediante la prueba de Chicuadrado. Revista Fuentes, 14(2), 5-18.

3. Ayala, F. E. B., Gómez, J. Q., \& León, E. A. (2011). Estudio de factibilidad del uso del biodiesel como fase contínua en lodos de perforación de emulsión inversa. Fuentes: El reventón energético, 9(1), 6 .
4. Banco de la República de Colombia (2016). Recuperado de: http://www.banrep.gov.co/ docum/Lectura_finanzas/pdf/re_200.pdf.

5. Barriga, F. (2009). El Petróleo y la Crisis Económica Mundial: una mezcla explosiva. Universidad San Francisco de Quito, pp. 36 - 43.

6. Besson, A., Burr, B., Dillard,S., Drake, E., Ivie,B., Ivie,C., ... Watson,G. (2000). On the cutting Edge. Oilfiled Review, vol. 20 (3), pp. 36 - 57.

7. Bone, G., Jamerson, C., Klanssen, J., Gray, J, Valliyapan, S., Baker, R.,Parra, M. (2013). Maximizing BHA Durability, Reliability, Turbodrill/Impregnated Bit Significantly Reduces Drilling Time in Granite Wash Laterals. Society of Petroleum Engineers, Amsterdam.

8. Brunton, G., Taylor, M., Crockett, R., DenBoer, D., Lund, J. y Fleming, C. (2014). Redifining PDC Bits. Oilfield Review, vol. 26 (2), pp. 48 -57.

9. Campana, D. E. A., \& Tapia, R. A. V. (2017). Evaluación cualitativa de la limpieza de hoyo en pozos de alta inclinación-alto desplazamiento en la Cuenca Oriente. Fuentes: El reventón energético, 15(2), 49-56.

10. CAMPETROL (2016) Boletín Ejecutivo de Taladros - Julio de 2016. Recuperado de: http://economicosysectoriales.campetrol.org/ informe-de-taladros/.

11. García, M., Quispe, C. y Ráez, L. (2003).Mejora Continua de la Calidad de los Procesos. Industrial Data, vol. 6 (1), pp. 89-94.

12. Gutiérrez, F. (2016). Conflictos y contextos regionales en la industria de los hidrocarburos en Colombia. Recuperado de: http://www.co.undp.org/ content/dam/colombia/docs/MedioAmbiente/ undp-co- Conflictos $\% 20 y \% 20$ contextos $\% 20$ regionales $\% 20$ en $\% 201 \mathrm{a} \% 20$ industria $\% 20$ de $\% 20$ loshidrocarburos\%20en\%20Colombia2016.pdf.

13. Grant, R. (2006). Dirección Estratégica, Georgetown: Thomson Reuters. 
14. Lopez, L.y Hernández, F. (2013). Recursos Humanos: La importancia de la motivación e incentivos para los trabajadores. Victoria: Universidad Autónoma de Tamaulipas.

15. Madjidi, A. (2001). Moving in the Right Direction. Middle East Reservoir Review, vol. 12 (2), pp. $48-55$.

16. Maella,P. (2010). ¿Cómo establecer una estrategia de recursos humanos eficaz? Barcelona: IESE Business School - Universidad de Navarra.

17. Portilla, H. E., Suárez, D. F., \& Corzo, R. (2012). Metodología para la optimización de parámetros de perforación a partir de propiedades geomecánicas. Revista Fuentes, 10(2).
18. Project Management Institute (2013). PMBOK Guide and Standards, Philadelphia: PMI.

19. Rickard, W., Bailey, A., Pahler, M. y Cory, S. (2014). Kymera Hybrid Bit Technology Reduces Drilling Cost. Thirty-Ninth Workshop on Geothermal Reservoir Engineering, Standford.

20. Schlumberger. (2002). SLB Media. Recuperado de: http://www.slb.com/ /media/Files/evaluation/ brochures/wireline_open_hole/geology/ubi br.pdf

21. Walt, A.,Cook, J., Bern, P., Carpenter,B., Hutchinson, M., Lovell, J., ... Rezmer-Cooper, I. (1998). Using Downhole Annular Pressure Measurements to Improve Drilling Performance. Oilfield Review, vol. 9 (4), pp. $40-55$.

Recepción: 13 de diciembre de 2017

Aceptación: 18 de abril de 2018 